\title{
Escala de Avaliação dos Estilos de Ensino: qualidades psicométricas dos dados
}

\author{
Orlanda Cruq. \\ Cecília Aguiar \\ Silvia Barros
}

\begin{abstract}
Resumo
Este trabalho apresenta uma escala de avaliação da qualidade dos comportamentos interactivos maternos. Embora este instrumento tenha sido utilizado no contexto de um projecto mais amplo que visa estudar os determinantes maternos da qualidade do envolvimento da criança, este artigo centra-se especificamente nas decisões metodológicas relativas à observação dos comportamentos interactivos maternos. A Escala de Avaliação dos Estilos de Ensino (EAEE) foi utilizada para observar os comportamentos interactivos de 120 mães de crianças em idade de creche, numa situação de jogo livre, seguida de arrumação dos brinquedos. Cada díade foi observada em três momentos diferentes. A análise das dimensões subjacentes, dos coeficientes de consistência interna e de acordo inter-observadores, bem como da consistência temporal dos dados, permite concluir que a EAEE é uma medida útil e adequada dos comportamentos interactivos de mães portuguesas em situações diádicas de jogo.

Palavras-Chave: Escala de avaliação; Comportamentos interactivos maternos; Jogo livre; Responsividade; Crianças de 1 a 2 anos.
\end{abstract}

\section{Teaching Styles Rating Scale: Data pscychometric properties}

\begin{abstract}
This paper presents a rating scale designed to capture the quality of maternal interaction behaviors. Although this instrument was used in the context of a broader research project, which intended to study maternal determinants of child's engagement quality, this article will specifically focus methodological decisions concerning the observation of mothers' interaction behaviours. The Teaching Styles Rating Scale (TSRS) was used to observe interaction behaviours of 120 mothers of toddlers in a free play situation followed by a cleanup instruction. Each dyad was observed in three different times. The examination of underlying dimensions, internal consistency, inter-rater agreement coefficients and temporal consistency data allow us to conclude that TSRS is a useful and adequate measure of Portuguese mothers interaction behaviors in dyadic play situations.

Keywords: Rating scale; Mothers' interaction behaviors; Free-play; Responsivity; Toddlers.
\end{abstract}

A interacção social pais-criança é o primeiro contexto de socialização de carácter diádico no seio do qual se geram oportunidades de desenvolvimento aos mais diversos níveis: cognitivo, emocional, social, self, etc. É no contexto desta interacção que flúem emoções de diferentes intensidades, regras e princípios convencionais e morais, conceitos físicos e sociais mais ou menos abstractos, etc., o que leva os investigadores a estabelecerem relações entre a qualidade da interacção pais-criança e o sentimento de auto-competência, o desenvolvimento moral, a competência cognitiva, linguística, social e de auto-regulação, para citar apenas alguns dos domínios do desenvolvimento da criança mais estudados.

Do ponto de vista metodológico, os investigadores têm utilizado diferentes métodos para aceder à realidade complexa das interacções sociais entre mães e filhos. Trata-se de traduzir, através de uma linguagem validada cientificamente, a complexidade das relações humanas, tendo porém a noção de que há dimensões importantes nessas relações que dificilmente serão captadas (Laosa, 1981).

Os primeiros estudos sistemáticos da relação mãe-criança surgiram a seguir à Segunda Guerra Mundial no âmbito do estudo do Fels Institute (Baldwin, 1948). De então para cá têm sido utilizadas situações de recolha de dados mais ou menos estruturadas, obtendo-se a informação de forma indirecta, através de entrevistas e questionários, ou de forma mais directa, através da observação. Estas metodologias podem ser colocadas num contínuo: num dos extremos situam-se os métodos mais indirectos - as entrevistas e os questionários - em que $\mathrm{O}$ investigador acede à realidade através das

Agradecemos às famílias que participaram neste estudo. Agradecemos igualmente aos restantes elementos da equipa que colaboraram na recolha de dados: Ana Isabel Pinto, Ana Susana Almeida, Leen Poppe e Maria da Paz Mascarenhas.

${ }^{1}$ Endereço para correspondência:

Faculdade de Psicologia e de Ciências da Educação da Universidade do Porto

Rua do Campo Alegre, no 1.021/1.055 - 4169-004 - Porto - Portugal

E-mail: orlanda@fpce.up.pt 
percepções e representações de indivíduos que funcionam como fonte de informação (em geral, os pais); no outro extremo situam-se os métodos mais directos - observacionais - no sentido em que o observador acede directamente à realidade sem ter de permeio outros agentes informativos, à excepção, naturalmente, da sua própria pessoa. A nossa opção metodológica encaixa-se nesta segunda possibilidade, recorrendo-se à observação directa da interacção diádica entre mãe e filho.

A opção pela metodologia de observação implica todo um conjunto de decisões, quer relativamente à situação na qual vai ser observada a interacção, quer relativamente à definição da unidade de análise. Por outro lado, deve ser considerado um conjunto de precauções a fim de salvaguardar a fidedignidade dos resultados observados. São estes os tópicos que abordaremos de seguida.

O local de observação (casa, escola, laboratório, etc.), os estímulos introduzidos (material, instruções) e o momento escolhido constituem o contexto no qual ocorrem os comportamentos. Este contexto pode ser definido por diferentes níveis de estruturação introduzidos pelo investigador. Quando a observação ocorre nos contextos naturais habitualmente frequentados pelas pessoas na sua rotina diária, o nível de estruturação introduzido é mínimo. Porém, quando se pretende estudar situações com algum grau de especificidade, com o objectivo de observar determinados comportamentos interactivos, torna-se necessário introduzir um nível superior de estruturação e escolher o cenário mais adequado, em termos de espaço, tempo, materiais e procedimento. Desde logo, a "representativeness and ecological validity of the conditions of observation deserve serious consideration" (Maccoby \& Martin, 1983, p. 18). As situações, independentemente de decorrerem em contexto natural ou laboratorial, devem estar relacionadas com as vivências das pessoas, sob pena de procedermos a observações que, mesmo rigorosas do ponto de vista metodológico, são artificiais.

Os comportamentos interactivos variam naturalmente em função dos cenários que os enquadram. Por vezes, os investigadores estão mais interessados em perceber as fontes de variação dos comportamentos interactivos associadas a esses cenários. Outras vezes, os investigadores estão mais interessados em perceber quais os "padrões" interactivos que caracterizam as pessoas, independentemente da variação situacional; procura-se assim uma consistência ao nível do padrão de interacção individual ou da díade. Obviamente, não é possível identificar um padrão interactivo consistente se a observação for realizada num momento temporal único. Levanta-se então a questão do número de vezes necessário para se obter uma representação adequada do padrão interactivo (Maccoby \& Martin, 1983). A observação em mais do que um momento é também pertinente se considerarmos que a presença do observador, enquanto elemento estranho à díade, vai ficando cada vez mais esbatida à medida que as situações se vão repetindo. Foi neste sentido que se orientou a nossa opção, já que utilizamos três momentos na recolha de dados.

Existem duas abordagens na delimitação do foco de observação e análise das interacções: a primeira é a microanálise das sequências comportamentais e a segunda é a análise dos comportamentos interactivos, não valorizando a sequência com que eles surgem. A primeira abordagem é especialmente pertinente quando se pretende perceber a natureza das contingências interactivas entre os elementos da díade e exige normalmente o recurso a registos videográficos. Não vamos entrar aqui em detalhe quanto a este tipo de análise porque não foi neste sentido que a nossa opção empírica se direccionou. Pelo contrário, focalizamo-nos nos comportamentos interactivos e, neste sentido, levantam-se algumas questões.

A primeira questão prende-se com o nível de proximidade que o nosso registo assume face aos dados comportamentais concretos. E aqui surgem duas possibilidades. Uma primeira possibilidade consiste em delimitar unidades comportamentais discretas, descrevê-las de forma cuidadosa através de comportamentos precisos e, de seguida, proceder a um registo de frequências dessas mesmas unidades, quer através de amostragens no tempo, quer através do registo de ocorrências. No final, poder-se-á calcular uma frequência global dos comportamentos, na qual os indivíduos podem ser comparados. Da mesma maneira, é possível construir $a$ posteriori um conjunto de medidas compósitas, através de metodologias estatísticas adequadas, que permitem uma leitura da realidade mais englobante, mais fácil e mais clara.

Porém, em vez de nos centrarmos no registo de unidades comportamentais simples, uma segunda possibilidade consiste em definir variáveis comportamentais cuja importância é quantificada através de uma escala de avaliação. Neste caso, a avaliação da importância destas variáveis na situação de observação concreta implica um julgamento genérico, não só da frequência com que surgem, mas também da sua duração e intensidade. Frequentemente são utilizados descritores que servem de ajuda ao observador.

Alguns investigadores consideram que as escalas de avaliação permitem obter representações mais válidas da realidade do que as medidas de frequência, porque permitem aos observadores fazer avaliações mais globais dos comportamentos observados. "Ratings can better capture the sensitivity of parental behavior and the context in which it occurs than do frequencies" (Rothbaum \& Crockenberg, 1995, p. 194). Por exemplo, 
se quisermos captar a responsividade da mãe aos interesses da criança é melhor dispor de uma avaliação global do que contar o número de vezes que a mãe segue as iniciativas da criança, visto que a responsividade da mãe aos interesses da criança é mais do que este comportamento específico. É mais proveitoso pensar a responsividade não como uma sequência de actos particulares, mas sim como uma disposição mediadora dos comportamentos interactivos da mãe. Neste sentido, as escalas de avaliação são mais adequadas. Esta é provavelmente a razão pela qual os estudos que utilizam escalas de avaliação apresentam correlações mais elevadas e melhores preditores (Schaefer, 1989).

A nossa opção caiu assim sobre a utilização de escalas de avaliação. Porém, convém ter presente que estas escalas, tendo um forte pendor inferencial, podem também ser mais influenciadas por impressões subjectivas e por condições não controladas, tais como os conhecimentos prévios e os preconceitos do observador.

Qualquer situação de observação levanta problemas em termos da descrição clara e objectiva das unidades de observação e do risco de subjectividade inerente ao observador. Neste sentido, é imprescindível recorrer ao acordo inter-observador a fim de demonstrar que estas unidades comportamentais possuem uma existência própria, que ultrapassa os pressupostos conceptuais e as características idiossincráticas de cada investigador individual.

\section{Decisões metodológicas assumidas neste estudo}

Qual a situação de observação dos comportamentos interactivos maternos por nós criada? Trata-se de uma situação de jogo livre, seguido de arrumação dos brinquedos, sujeita aos seguintes condicionamentos:

1. Espaço disponibilizado na creche frequentada pela criança, sob condição de não haver interrupções.

2. Momento temporal de maior disponibilidade para as mães, em geral coincidindo com a chegada ou partida das crianças à creche.

3. Duração total de 15 minutos, sendo 10 minutos ocupados com o jogo livre e 5 minutos dedicados à arrumação do material.

4. O material era constituído por um conjunto de brinquedos e jogos adequados às faixas etárias entre o 1 e os 3 anos; foi ainda incluído um jogo considerado mais difícil para que as crianças mais velhas estivessem em igualdade de circunstâncias em relação às mais novas (no que diz respeito ao grau de dificuldade dos brinquedos); todos os objectos estavam guardados dentro de uma caixa plástica transparente. 5. A instrução apresentada às mães consistia em: (1) brincar com os filhos como se estivessem em casa; (2) quando lhes fosse dado um sinal, pedir à criança para arrumar os brinquedos dentro da caixa.

6. A observação foi feita em três dias distintos, com intervalos de tempo muito variáveis, desde 1 até 99 dias $(92,5 \%$ das sessões foram realizadas num intervalo de 30 dias). Foram utilizadas sempre as mesmas condições de observação.

7. Todas as sessões foram gravadas em vídeo, com o prévio consentimento das mães.

Por quê uma situação de jogo livre, seguida de instrução para arrumar? Pensamos que se trata de uma situação com razoável validade ecológica, já que a grande maioria das mães nas sociedades ocidentais brincam frequentemente com os seus filhos desta idade. Por outro lado, as mães começam a incluir a criança na tarefa de arrumação dos brinquedos. A situação de jogo livre tem sido utilizada em múltiplas investigações em que se procura perceber os determinantes maternos do desenvolvimento da criança, quer na área cognitiva, quer na competência de auto-regulação (Kochanska, Coy \& Murray, 2001). A situação de arrumação dos brinquedos fornece à mãe um argumento para introduzir alguma directividade na relação com a criança que, de outra forma, não teria tanta possibilidade de surgir na situação de jogo; permite observar como a mãe gere a situação de colocar a criança a fazer algo que à partida não lhe é tão interessante, situação esta bastante comum no quotidiano de crianças desta faixa etária.

A Escala de Avaliação dos Estilos de Ensino (EAEE), inicialmente desenvolvida por McWilliam, Scarborough, Bagby \& Sweeney (1998, Teaching Styles Rating Scale, TSRS) para observação dos comportamentos interactivos dos educadores, foi adaptada tendo em vista a sua aplicação à observação dos comportamentos interactivos das mães. Neste artigo serão apresentados dados relativos à estrutura factorial, fidelidade (consistência interna), acordo inter-codificador e consistência temporal das observações realizadas.

\section{Método}

\section{Participantes}

Foram seleccionadas de forma aleatória 120 díades mãe-criança a partir de 30 salas de creche. $\mathrm{Na}$ Tabela 1 apresentam-se sucintamente algumas características das crianças e mães participantes. 
Tabela 1 - Características dos participantes

\begin{tabular}{cccc}
\hline & $\mathrm{M}$ & $\mathrm{DP}$ & Variação \\
\hline Características da mãe (n=120) & & 5,3 & $18-43$ \\
Idade (anos) & 30,9 & 4,47 & $0-18$ \\
Nível educativo (anos) & 10,83 & & $14-49$ \\
\hline Características da criança (n $=120)$ & & & 7,07 \\
(60 rapazes e 60 raparigas) & 26,19 & 9,87 & $79,45-132,35$ \\
Idade cronológica (meses) & 103,56 & & \\
Quociente desenvolvimental & & & \\
\hline
\end{tabular}

\section{Instrumento}

A Escala de Avaliação dos Estilos de Ensino foi aplicada com base nos registos videográficos dos comportamentos das mães, realizados no contexto da situação de interacção lúdica padronizada já descrita.

A EAEE é composta por 18 itens, organizados em duas sub-escalas: a sub-escala dos comportamentos de ensino (7 itens) e a sub-escala do afecto (11 itens). $\mathrm{Na}$ primeira sub-escala é utilizada uma escala de avaliação de 7 pontos com descritores genéricos nos valores ímpares, enquanto que na sub-escala do afecto é utilizada uma escala de avaliação de 5 pontos, com descritores específicos também nos valores ímpares. Os itens aparecem listados nas Tabelas 2 e 3.

\section{Procedimento}

A situação lúdica de interacção mãe-criança atrás descrita teve lugar após uma entrevista à mãe em que se aplicava um questionário para avaliação de características de temperamento e competência da criança e que permitia, ao mesmo tempo, promover a criação de uma relação de confiança do investigador com a mãe. A observação das interacções diádicas foi repetida ao longo de três sessões que ocorreram em momentos temporais distintos.

No decorrer do procedimento de cotação das sessões, realizaram-se verificações inter-observadores do acordo em $25 \%$ das sessões de observação avaliadas com a EAEE (um segundo observador foi utilizado num total de 90 sessões). Todas as crianças foram avaliadas através das Escalas de Desenvolvimento Mental de Ruth Griffiths (1986).

\section{Resultados}

\section{Estrutura factorial}

Foi realizada uma análise de componentes principais, seguida de uma rotação varimax, com o objectivo de determinar a estrutura factorial da escala em estudo e reduzir os dados iniciais. As componentes resultantes são apresentadas na Tabela 2 .

Tabela 2 - Estrutura factorial dos itens

\begin{tabular}{lccc}
\hline Comportamentos interactivos & Responsividade & Ensino activo & Não responsividade \\
\hline Redireccionamento & $\mathbf{- 0 , 8 4}$ & 0,07 & 0,21 \\
Directividade & $\mathbf{0 , 8 3}$ & $-0,16$ & $-0,19$ \\
Responsividade face aos interesses & $\mathbf{0 , 8 1}$ & 0,32 & $-0,19$ \\
Tom & $\mathbf{0 , 7 9}$ & 0,15 & 0,14 \\
Expressão negativa & $\mathbf{0 , 6 7}$ & 0,22 & 0,24 \\
Responsividade emocional & $\mathbf{0 , 6 6}$ & 0,58 & 0,07 \\
Reconhecimento & $\mathbf{0 , 6 2}$ & 0,26 & $\mathbf{- 0 , 4 2}$ \\
Consistência da interacção & $\mathbf{0 , 6 1}$ & 0,42 & 0,01 \\
Nível de actividade & $-0,31$ & $\mathbf{0 , 8 1}$ & 0,10 \\
Elaboração & 0,38 & $\mathbf{0 , 7 2}$ & $-0,17$ \\
Adequação desenvolvimental & 0,51 & $\mathbf{0 , 7 1}$ & $-0,10$ \\
Desencadeamento da resposta & 0,23 & $\mathbf{0 , 6 9}$ & $-0,24$ \\
Expressão positiva & 0,37 & $\mathbf{0 , 6 6}$ & 0,29 \\
Elogio & 0,04 & $\mathbf{0 , 5 6}$ & 0,20 \\
Informação & 0,01 & $\mathbf{0 , 5 1}$ & $-0,27$ \\
Responsividade física & $-0,13$ & 0,04 & $\mathbf{0 , 7 7}$ \\
Introdução & $-0,14$ & $-0,11$ & $\mathbf{0 , 7 0}$ \\
Envolvimento visual & 0,26 & 0,09 & 0,35
\end{tabular}


O factor 1, responsável por 37,1\% da variância, é saturado por itens que traduzem não só uma atitude relacional positiva face à criança, mas também uma atitude responsiva. $\mathrm{O}$ segundo factor integra maioritariamente itens que supõem uma atitude de estimulação activa e de reforço das respostas da criança. Finalmente, o factor 3 inclui um pequeno número de itens que apontam no sentido de uma forte orientação da acção da criança, sem reconhecimento das suas necessidades.

\section{Fidelidade dos dados}

No sentido de determinar a fidelidade dos factores encontrados foram calculados o acordo interobservadores e o coeficiente weighted kappa. $\mathrm{O}$ acordo inter-observadores, com diferenças de um valor, variou entre $89 \%$ e $99 \%$ em todos os itens da escala. A média de acordo inter-observadores foi de $96 \%$ para o primeiro e segundo factores e de $95 \%$ para o terceiro factor. Os valores médios obtidos para o coeficiente weighted kappa foram 0,43 para o factor 1, 0,41 para o factor 2 e de 0,37 para o factor 3 .

No que se refere à consistência interna dos factores obtidos, determinada com base no coeficiente alfa de Cronbach, apurou-se um alfa de 0,91 para o primeiro factor, um alfa de 0,87 para o segundo factor e de 0,59 para o terceiro factor.
Ainda no que se refere à fidelidade dos dados, o cálculo do coeficiente de correlação intraclasses permitiu verificar que a utilização de dados relativos a uma única sessão permitiria obter dados em que a proporção de variância associada a diferenças entre participantes seria, em média, 0,43 (o que significa que, em média, $57 \%$ da variância é atribuível a outros factores que não às características dos próprios participantes). A utilização da média de três sessões determinou um aumento considerável da proporção de variância associada a diferenças entre participantes $(0,68)$. Assim, neste estudo, a utilização da média dos resultados obtidos nas três sessões disponíveis permitiu reduzir a proporção de variância devida a outros factores (nomeadamente a diferenças entre observadores e a erro de medida) para $32 \%$.

Foram ainda calculadas correlações no sentido de determinar a associação entre os resultados obtidos nas três sessões (ao nível dos itens da escala). Estes resultados permitem obter informações relativas à consistência ao longo do tempo dos dados obtidos com a EAEE. O significado prático dos resultados foi interpretado de acordo com as convenções definidas por Cohen (1992): um $r$ de 0,10 foi considerado pequeno e revela uma associação fraca; um $r$ de 0,30 foi considerado médio e revela uma associação moderada, e um $r$ de 0,50 foi interpretado como grande, revelando uma associação forte.

Tabela 3 - Correlações entre os resultados obtidos nas três sessões

\begin{tabular}{|c|c|c|c|}
\hline Item & 1 vs. 2 & 2 vs. 3 & 1 vs. 3 \\
\hline Redireccionamento & $0,665^{* *}$ & $0,582^{* *}$ & $0,534^{* *}$ \\
\hline Introdução & $0,276^{* *}$ & $0,296^{* *}$ & $0,251^{*}$ \\
\hline Elaboração & $0,572^{* *}$ & $0,559^{* *}$ & $0,641^{* *}$ \\
\hline Desencadeamento da Respostas & $0,399^{* *}$ & $0,387^{* *}$ & $0,234^{*}$ \\
\hline Informação & $0,237^{* *}$ & $0,385^{* *}$ & 0,071 \\
\hline Reconhece & $0,389^{* *}$ & $0,507^{* *}$ & $0,354^{* *}$ \\
\hline Elogio & $0,394^{* *}$ & $0,368^{* *}$ & $0,474^{* *}$ \\
\hline Nível de Actividade & $0,585^{* *}$ & $0,570^{* *}$ & $0,497^{* *}$ \\
\hline Expressão Positiva & $0,596^{* *}$ & $0,510^{* *}$ & $0,536^{\text {** }}$ \\
\hline Expressão Negativa & $0,424^{* *}$ & $0,478^{* *}$ & $0,373^{* *}$ \\
\hline Envolvimento visual & $0,208^{*}$ & $0,219^{*}$ & $0,323^{* *}$ \\
\hline Responsividade física & $0,227^{*}$ & 0,170 & $0,483^{* *}$ \\
\hline Responsividade Emocional & $0,441^{* *}$ & $0,510^{* *}$ & $0,446^{* *}$ \\
\hline Consistência de interacção & $0,286^{* *}$ & $0,234^{*}$ & $0,278^{* *}$ \\
\hline Responsividade aos interesses da criança & $0,601^{* *}$ & $0,522^{* *}$ & $0,489^{* *}$ \\
\hline Directividade & $0,471^{* *}$ & $0,532^{* *}$ & $0,379^{* *}$ \\
\hline Tom & $0,518^{* *}$ & $0,629^{* *}$ & $0,566^{* *}$ \\
\hline Adequação Desenvolvimental & $0,577^{* *}$ & $0,482^{* *}$ & $0,523^{* *}$ \\
\hline
\end{tabular}
${ }^{*} p<0,05 ;{ }^{* *} p<0,01$

Como se pode verificar na Tabela 3, a magnitude das associações entre diferentes sessões varia de item para item. Note-se, contudo, que todas as correlações calculadas foram estatisticamente significativas. As mães manifestaram maior consistência no que diz respeito Psico-USF, v. 9, n. 2, p. 165-171, Jul./Dez. 2004 aos comportamentos de redireccionamento, elaboração, expressão positiva, tom, responsividade em relação aos interesses da criança e adequação desenvolvimental. Nestes itens, as correlações entre sessões, interpretadas neste estudo como indicadores de estabilidade e 
consistência ao longo do tempo, indicaram, de uma forma geral, associações fortes. Os itens em que se verificou menor consistência entre sessões relacionavam-se com a introdução de novas actividades, fornecimento de informação não relacionada com as actividades, envolvimento visual, responsividade física e consistência das interacções (nestas dimensões, a magnitude de associação entre sessões foi, em média, pequena). Nos itens restantes, verificou-se uma consistência entre observações que se pode considerar moderada.

\section{Discussão}

Os factores obtidos com base na EAEE correspondem a conceitos compatíveis com a literatura dominante na área dos comportamentos educativos maternos. O primeiro conceito é o de responsividade materna, associado a um clima afectivo positivo. A responsividade implica perceber as pistas ou sinais expressos pela criança, interpretá-los adequadamente e seleccionar uma resposta pronta e contingente (Bornstein \& TamisLe-Monda, 1997). A resposta responsiva é adequada no tipo, tempo e intensidade às necessidades e desejos da criança, incluindo as suas necessidades desenvolvimentais. A literatura tem consistentemente demonstrado que a responsividade está associada a bons indicadores desenvolvimentais (Kochanska, 1997; Kochanska \& Aksan, 1995; Landry, Smith, Swank, Assel \& Vellet, 2001; Lewis, 1993; Londerville \& Main, 1981). Pelo contrário, a ausência de responsividade na infância está associada a resultados negativos em indicadores de desenvolvimento social (Landry e colaboradores, 2001) a comportamentos de externalização (Shaw, Keenan \& Vondra, 1994) e a problemas comportamentais na idade escolar (Wakschlag \& Hans, 1999).

É possível identificar no comportamento interactivo materno outras dimensões positivas, como é o caso do ensino activo (Bornstein \& Tamis-Le-Monda, 1997; Wakschlag \& Hans, 1999). Esta dimensão referese à atitude activa positiva assumida pela mãe quando ensina, estrutura e traça limites, enfatizando o uso da linguagem, o afecto positivo e a demonstração do uso de brinquedos ou outros materiais (Wakschlag \& Hans, 1999). Assim, a situação de interacção com a criança pode ser abordada pelas mães como uma oportunidade para, de forma relaxada e gratificante, se envolverem com os seus filhos, respondendo aos seus interesses e emoções, ou como uma oportunidade para ensinar, estimular e promover o desenvolvimento da criança. No primeiro caso predomina a atitude responsiva, enquanto que no segundo caso predomina a atitude de ensino activo.

Os resultados relativos às características de fidelidade dos dados indicam que a EAEE constitui um instrumento útil na determinação dos estilos de ensino das mães. Efectivamente, através desta escala de avaliação, é possível obter dimensões de comportamento interactivo conceptualmente coerentes, com níveis satisfatórios de acordo inter-observadores e níveis de acordo devido ao acaso que se podem considerar aceitáveis neste domínio de investigação. O cálculo do coeficiente alfa de Cronbach revela bons níveis de consistência interna nos dois primeiros factores, sugerindo que cada um deles mede, individualmente, um único constructo. $\mathrm{O}$ terceiro factor extraído não apresenta níveis de consistência interna satisfatórios, facto que poderá estar associado ao limitado número de itens.

Um contributo metodológico deste trabalho que se poderá revelar útil para outros investigadores baseia-se nos resultados do coeficiente de correlação intraclasses. Os dados obtidos através deste coeficiente sugerem que a combinação de cotações múltiplas produz dados de maior qualidade psicométrica (McGraw \& Wong, 1996), ou seja, é possível obter dados mais fidedignos realizando mais do que uma sessão de observação e combinando os resultados obtidos em diferentes sessões. Investigação adicional no sentido de determinar quantas sessões são necessárias para obter níveis adequados de fidelidade e estabilidade seriam de grande utilidade (nomeadamente, para informar a elaboração de desenhos experimentais).

A análise das correlações entre os resultados obtidos nas três sessões disponíveis revela níveis aceitáveis de consistência e estabilidade ao longo do tempo. Naturalmente, a consistência dos comportamentos interactivos maternos varia de comportamento para comportamento. As variações observadas nas diferentes sessões reforçam a idéia avançada anteriormente no sentido de realizar múltiplas sessões de observação para obter uma imagem mais estável do perfil interactivo de cada mãe ou adulto que interage com a criança.

A observação do comportamento humano continua a colocar-nos enormes desafios. Esperamos ter demonstrado que esta escala de observação dos comportamentos interactivos das mães preenche os requisitos de rigor científico necessários à continuação da investigação, desde que sejam cumpridas exigências metodológicas relacionadas com a preparação dos observadores e a estabilidade temporal dos resultados.

\section{Referências}

Baldwin, A. L. (1948). Socialization and parent-child relationship. Child Development, 19, 127-136.

Bornstein, M. H. \& Tamis-Le-Monda, C. S. (1997). Maternal responsiveness and infant mental abilities: Specific predictive relations. Infant Behavior and Development, 20, 283-296.

Cohen, J. (1992). Quantitative methods in Psychology: A power primer. Psychological Bulletin, 112, 155-159. 
Griffiths, R. (1996). The Griffith's mental development scales, from birth to 2 years. Manual. The Test Agency.

Kochanska, G. (1997). Multiple pathways to conscience for children with different temperaments: From toddlerhood to age 5. Developmental Psychology, 33, 228-240.

Kochanska, G. \& Aksan, N. (1995). Mother-child mutually positive affect, the quality of child compliance to requests and prohibitions, and maternal control as correlates of early internalization. Child Development, 66, 236-254.

Kochanska, G., Coy, K. C. \& Murray, K. T. (2001). The development of self-regulation in the first four years of life. Child Development, 72, 1091-1111.

Landry, S. H., Smith, K. E., Swank, P. R., Assel, M. A. \& Vellet, S. (2001). Does early responsive parenting have a special importance for children's development or is consistency across early childhood necessary? Development Psychology, 37, 387-403.

Laosa, L. (1981). Maternal behaviour: Sociocultural diversity in modes of family interaction. Em R. W. Henderson (Org.). Parent-child interaction. Theory, research, and prospects. New York: Academic Press.

Lewis, M. D. (1993). Early socioemotional predictors of cognitive competency at 4 years. Developmental Psychology, 29, 1036-1045.

Londerville, S. \& Main, M. (1981). Security of attachment, compliance, and maternal training methods in the second year of life. Developmental Psychology, 17, 289-299.
Maccoby, E. E. \& Martin, J. A. (1983). Socialization in the context of the family: Parent-child interaction. Em P. H. Mussen \& E. M. Hetherington (Orgs.). Handbook of child psychology: Vol. 4. Socialization, personality and social development (pp. 1-101). New York: John Wiley \& Sons.

McGraw, K. O. \& Wong, S. P. (1996). Forming inferences about some intraclass correlation coefficients. Psychological Methods, 1, 30-46.

McWilliam, R. A., Scarborough, A. A., Bagby, J. H. \& Sweeney, A. L. (1998). Teaching Styles Rating Scale. Chapel Hill, NC: Frank Porter Graham Child Development Center. University of North Carolina at Chapel Hill.

Rothbaum, F. \& Crockenberg, S. (1995). Maternal control and two-year-olds' compliance and defiance. International Journal of Behavioral Development, 18, 193-210.

Schaefer, E. S. (1989). Dimensions of mother-infant interaction: Measurement, stability and predictive validity. Infant Behavior and Development, 12, 379-393.

Shaw, D. S., Keenan, K. \& Vondra, J. I. (1994). Developmental precursors of externalizing behaviour: Ages 1 to 3. Developmental Psychology, 30, 355-364.

Wakschlag, L. S. \& Hans, S. L. (1999). Relation of maternal responsiveness during infancy to the development of behaviour problems in high-risk youths. Developmental Psychology, 35, 569-579.

Recebido em novembro de 2004 Reformulado em novembro de 2004 Aprovado em dezembro de 2004

Sobre as autoras:

Orlanda Cruz é doutora em Psicologia do Desenvolvimento, professora da Faculdade de Psicologia e de Ciências da Educação da Universidade do Porto (FPCE-UP), Portugal, e Coordenadora do Mestrado em Intervenção Psicológica com Crianças e Adolescentes.

Cecília Aguiar é psicóloga, mestra e doutoranda em Psicologia pela Faculdade de Psicologia e de Ciências da Educação da Universidade do Porto (FPCE-UP), Portugal, colaborando como investigadora no Centro de Psicologia da Universidade do Porto.

Sílvia Barros é psicóloga e doutoranda em Psicologia pela Faculdade de Psicologia e de Ciências da Educação da Universidade do Porto (FPCE-UP), Portugal, colaborando como investigadora no Centro de Psicologia da Universidade do Porto. 


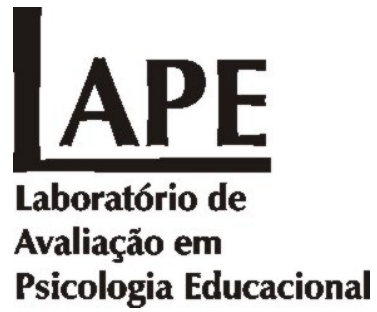

Programa de Pós-Graduação Stricto Sensu em Psicologia da Universidade São Francisco

Estudo de instrumentos de avaliação de processos cognitivos e afetivos envolvidos em situações de aprendizagem nos diversos contextos de escolarização. Envolve a participação de doutorandos, mestrandos e bolsistas de Iniciação Científica.

Professores responsáveis:

Dra. Acácia Aparecida Angeli dos Santos acacia.santos@saofrancisco.edu.br

Dr. Fermino Fernandes Sisto

fermino.sisto@saofrancisco.edu.br

Dra. Maria Cristina Rodrigues Azevedo Joly maria.joly@saofrancisco.edu.br 